\title{
O Significado da Abstinência do Álcool na Cardiomiopatia Alcoólica com Disfunção Ventricular Moderada
}

\author{
Francisco Manes Albanesi Fo ,Márcia Bueno Castier, Silvia Helena Cardoso Boghossian, \\ Tatiana Tavares da Silva
}

\author{
Rio de Janeiro, RJ
}

\begin{abstract}
Objetivo - Avaliar o papel da abstenção alcoólica, no periodo de 12 meses, na cardiomiopatia alcoólica (CMA) com disfunção ventricular de grau moderado, em pacientes tratados com esquema anticongestivo.

Métodos - Estudo observacional prospectivo com 20 pacientes com CMA, $9(45 \%)$ na classe funcional (CF) II e $11(55 \%)$ na CF III, 16 (80\%) homens, predomínio de negros $(55 \%)$, de 35 a $56(x=45)$ anos, com consumo pesado de álcool (>80g etanol/dia), por periodo de 51 a $112(x=85)$ meses, que concordaram, inicialmente, em participar de programa de apoio, com psicoterapia de grupo, além do acompanhamento clínico com realização de exames não invasivos, antes do início do programa e após 12 meses da terapia, e foram divididos em dois grupos, o 1 (G-I) constituido pelos que atingiram a abstinência e o 2 (G-II) pelos não abstêmios.

Resultados - Após 12 meses, 11 (55\%) pacientes permaneciam em terapia de apoio, $8(72,72 \%)$ no G-I, enquanto os 9 (45\%) que não se mantiveram no programa, apenas $2(22,22 \%)$ tinham logrado abstinência $(G-I)$. Ao fim da avaliação, alcançamos número igual de pacientes entre os grupos. Comparando os grupos observamos: a) menores valores médios dos diâmetros sistólico (DSVE) e diastólico (DDVE) do ventrículo esquerdo no $G$-I; $b$ ) maior número de internações no G-II (3) em relação ao G-I (1); c) na evolução clínica, maior número de pacientes do $G$-I, entre os que apresentaram melhoras (3 G-Ie 1 G-II) e que permaneceram inalterados (6 G-I e 3 G-II), além do maior número de pioras entre o G-II (I G-I e 5 G-II).

Conclusão - Apesar do desejo inicial favorável, a abstinência só foi obtida em $50 \%$ dos pacientes com CMA e disfunção ventricular moderada, porém, quando alcançada, apresentou melhor evolução (melhoras + inalterados $=90 \%$ ), com maior redução do DSVE e do DDVE $(p<0,001)$, devendo sempre ser perseguida mesmo na presença de moderada disfunção ventricular.
\end{abstract}

Palavras-chave: cardiomiopatia alcoólica, abstinência, evolução

\section{The Significance of Alcohol Abstinence in Alcoholic Cardiomyopathy with Moderate Left Ventricular Dysfunction}

Purpose - To evaluate the role of a 12 month alcohol abstinence period in patients with moderate left ventricular dysfunction treated with anticongestive therapy.

Methods - Prospective observational study with 20 patients with alcoholic cardiomyopathy (ACM), 9 $(45 \%)$ in functional class (FC) II and 11 (55\%) in FC III, 16 (80\%) men, mostly black (55\%), from 35 to 56 $(x=45)$ years old, heavy alcohol users $(>80 \mathrm{~g}$ ethanol for 51 to $112(x=88)$ months. At the beginning, all agreed to participate with psychotherapy and clinical evaluation. After 12 months, they were divided in $G-I$, formed by those who remained abstemious and G-II of non-abstemious.

Results - After 12 months, among the 11(55\%) who remained in psychotherapy, 8 were in G-I, among those who did not $9(45 \%)$, only $2(22,22 \%)$ remained abstemious $(G-I)$. At the end of the evaluation period, both groups had the same number of patients. Comparing them, we observed: a) lower mean systolic and diastolic left ventricular diameters in $G-I ; b)$ more hospitalizations in G-II (3); c) more patients with stable or better clinical evolution in G-I.

Conclusion - Despite the initial will, only 50\% reached abstinence. When it was reached, patients had a better evolution in left ventricular systolic diameter and abstinence should always be tried even in the presence of moderate left ventricular dysfunction.

Key-words: alcoholic cardiomyopathy, abstinence, evolution

Arq Bras Cardiol, volume 71 (n 6), 781-785, 1998

Faculdade de Ciências Médicas da Universidade do Estado do Rio de Janeiro Correspondência: Francisco Manes Albanesi $F^{o}-$ Av. 28 de Setembro, $77-2^{\circ}$. 20551- 030 - Rio de Janeiro, RJ

Recebido para publicação em 22/7/98

Aceito em 14/10/98
Foi Walshe ${ }^{1}$,em 1873, o primeiro a chamar atenção para o papel adverso exercido pelo álcool no coração. Entretanto, somente após o relato de Graham Steel ${ }^{2}$, em 1893, demonstrando em 25 casos de insuficiência cardíaca (IC) a as- 
sociação com o alcoolismo crônico, é que esta relação passou a ser aceita, porém com reservas pelos médicos. A partir da segunda metade deste século, ganhou maior difusão na clínica ${ }^{3-6}$. Entre os 673 pacientes consecutivos com IC congestiva estudados no período de 1982 a 1991, noJohns Hopkins Hospital, a causa mais freqüente foi a cardiomiopatia dilatada com $46,5 \%$, estando o álcool presente em 23 $(3,4 \%)$ pacientes, em indivíduos que consumiam mais do que $224 \mathrm{~g} /$ dia de etanol, por mais de seis meses ou em tratamento do alcoolismo? .

O elemento de maior significado no tratamento da cardiomiopatia alcoólica (CMA) é a abstinência, que deve ser perseguida em qualquer fase da agressão $0^{3-6,8-11}$. Alguns autores pregam a proibição total do consumo de álcool, não se admitindo sequer o seu uso social, pois deprime ainda mais o miocárdio, precipitando arritmias ${ }^{8-12}$. Recentemente, publicação do Serviço de Saúde Pública dos Estados Unidos da América aconselha desencorajar o seu consumo, sendo que naqueles onde for impossível alcançar a abstinência ou naqueles com pequeno grau de disfunção miocárdica, permitir o uso de dose única diária $(<28 \mathrm{~g} \text { de etanol } / \mathrm{dia})^{13}$. Assim, resolvemos avaliar o significado da abstinência na evolução da CMA, em portadores de disfunção miocárdica de grau moderado, que aceitaram participar espontaneamente de protocolo, onde a meta a ser alcançada era a retirada total do álcool, com terapia de apoio psicoterápico, em centro terciário na cidade do Rio de Janeiro.

\section{Métodos}

Foram avaliados, por estudo observacional prospectivo, 20 pacientes com CMA, que apresentavam disfunção ventricular de grau moderado, encontrando-se em tratamento ambulatorial, e que concordaram (consentimento escrito), após conhecer a proposta do estudo, em iniciar terapia de apoio psicológico regular, por período de 12 meses, visando se livrarem do vício do consumo excessivo do álcool, com o objetivo de verificar o papel da abstinência alcoólica na melhora, manutenção ou agravamento da disfunção miocárdica.

Foram excluídos do estudo os portadores de hipertensão arterial (complicação freqüente entre os que consomem excesso de álcool), os que apresentavam quadro anterior de angina do peito e passado de infarto do miocárdio, afastando algumas causas que, associadas ao consumo de álcool, poderiam ocasionar agressão miocárdica.

Eram 16(80\%) homens, comidades entre 35 a 56 $(x=45)$ anos, com predomínio de negros (55\%), com consumo considerado pesado de álcool e $>80 \mathrm{~g}$ de etanol/dia, segundo os critérios de Burch e Gilles ${ }^{8}$, por período de 51 a 112 ( $\mathrm{x}=85$ ) meses, avaliados segundo a classe funcional (CF) da New York Heart Association, 9 (45\%) na CF II e 11 (55\%) na CF III, mantidos em seguimento ambulatorial, em uso de terapia anticongestiva com digital, diurético (furosemida ou tiazídico) e inibidor da enzima conversora, otimizados em suas dosagens.

A disfunção ventricular esquerda foi avaliada por meios clínicos, eletrocardiográficos e medidas ecocardio- gráficas, sendo empregados os diâmetros diastólico do ventrículo esquerdo (DDVE) e sistólico do ventrículo esquerdo (DSVE); a dimensão do átrio esquerdo (AE) e do percentual de encurtamento circunferencial do ventrículo esquerdo (VE) - \% ES, comparando-se suas medidas antes e após a introdução do programa de apoio psicológico.

Os pacientes foram divididos em quatro subgrupos de cinco, constituindo os subgrupos de terapia de apoio, que era semanal, realizada em horário de comum acordo entre os seus participantes e terapeutas. Ao lado desse apoio, continuaram o acompanhamento ambulatorial a cada dois meses, ou quando necessário, sendo avaliados por exames clínico e complementar não invasivo (eletrocardiograma e ecocardiograma), comparando-se os achados antes do inicio da terapia de apoio e após 12 meses. Os que lograram atingir abstinência constituíram o grupo 1 (G-I) e os que permaneceram com hábitos alcoólicos o grupo 2 (G-II).

Ao término do estudo foram submetidos a teste de avaliação, visando conhecer: se consumiram ou não álcool (quantidades e motivos que o levaram a usá-lo), seus sintomas, sua experiência com a supressão da droga e suas reações quando mantidos no programa, e naqueles que abandonaram a terapia, as causas que motivaram essa decisão.

\section{Resultados}

Após transcorridos os 12 meses, permaneciam no programa de terapia de apoio 11 (55\%) pacientes, tendo oito logrado a abstinência e três que continuavam a consumir bebidas alcoólicas com freqüência, mesmo com a permanência no programa de psicoterapia. Entre os $9(45 \%)$ pacientes que abandonaram o programa de apoio, dois $(22,22 \%)$ tinham atingido a abstinência, porém o agente de transformação não havia sido a psicoterapia, mas sim o ingresso em nova seita religiosa que, entre outras metas, combatia também o alcoolismo (fig. 1). $\mathrm{O}$ abandono ocorreu entre o $3^{\circ} \mathrm{e}$ $10^{\circ}(\mathrm{x}=6,2)$ mês, tendo sido a causa mais freqüente, a vergonha pela mentira imposta aos companheiros do grupo psicoterápico, uma vez que estavam consumindo álcool e diziam que estavam se abstendo, reconhecendo que a abstinência era ato voluntário, que não conseguiam atingir, por não ultrapassarem os motivos que geraram o seu vício.

Ao término dos 12 meses alcançamos igual número de pacientes entre os grupos (fig. 2), porém, entre os que lograram abstenção (G-I), 3 (30\%) tinham apresentado melhoras na função ventricular, $6(60 \%)$ permaneciam inalterados e apenas $1(10 \%)$ tinha piorado. No entanto, entre os que não alcançaram a abstinência (G-II), apenas $1(10 \%)$ apresentou melhoras da função, $4(40 \%)$ permaneceram inalterados e 5 (50\%) pioraram a função ventricular. O único caso do G-II, apresentando melhoras, era o paciente que permaneceu no grupo de apoio e que passou a consumir menor volume de etanol por semana.

No julgamento final do protocolo, após o término do estudo utilizando os dados clínicos e os exames complementares, encontramos os seguintes achados. O eletrocardiograma evidenciou o aparecimento de um caso de fibri- 


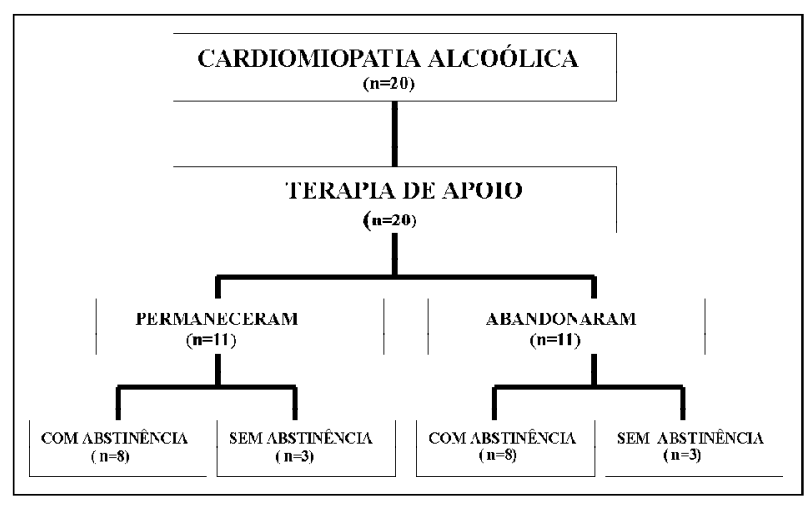

Fig. 1 - Resultados após os 12 meses do programa de apoio psicológico em 20 pacientes com cardiomiopatia alcoólica.

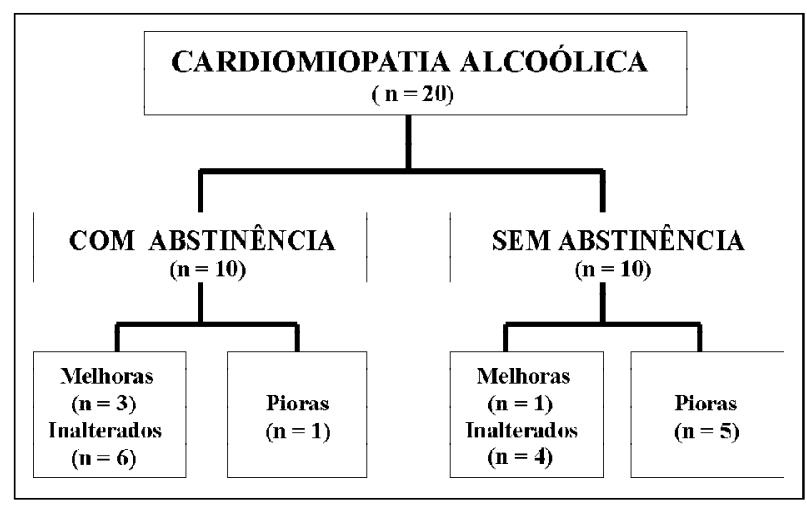

Fig. 2 - Evolução clínica entre os 20 pacientes com cardiomiopatia alcoólica submetidos a 12 meses do programa de apoio psicológico.

lação atrial no G-II, em paciente que apresentou piora do estado contráctil. Observamos redução no número de extrasístoles no G-I, porém sem diferença estatística com o G-II. O ecocardiograma (tab. I) revelou que o G-I apresentou redução significativa ( $\mathrm{p}<0,001)$ dos DSVEe DDVE, em relação aos valores obtidos antes do início do apoio psicológico, mostrando o papel desempenhado pela abstenção do álcool. Tivemos no período $4(20 \%)$ hospitalizações, sendo uma no G-I e 3 no G-II, todas em pacientes com piora do quadro clínico. Na avaliação do conjunto, observamos maior núme-

\begin{tabular}{|c|c|c|c|c|}
\hline \multicolumn{5}{|c|}{$\begin{array}{l}\text { Tabela I - Valores médios das medidas das câmaras esquerdas e } \\
\text { de função em pacientes com cardiomiopatia alcoólica, comparan- } \\
\text { do o início do estudo e a presença (G-I) ou não (G-II) da } \\
\text { abstinência alcoólica }\end{array}$} \\
\hline & \multirow[t]{2}{*}{ Início } & \multicolumn{3}{|c|}{ Término } \\
\hline & & G- I & G-II & $\mathrm{p}$ \\
\hline $\operatorname{DDVE}(\mathrm{cm})$ & 6,5 & 6,23 & 6,88 & $<0,001$ \\
\hline $\operatorname{DSVE}(\mathrm{cm})$ & 5,4 & 5,11 & 5,47 & $<0,001$ \\
\hline $\mathrm{AE}(\mathrm{cm})$ & 3,81 & 3,80 & 3,94 & $\mathrm{~ns}$ \\
\hline$\% \operatorname{ES}(\%)$ & 16,98 & 18,55 & 17,95 & $\mathrm{~ns}$ \\
\hline \multicolumn{5}{|c|}{$\begin{array}{l}\text { DDVE- diâmetro diastólico do ventrículo esquerdo; DSVE- diâmetro } \\
\text { sistólico do ventrículo esquerdo; AE- átrio esquerdo; \% ES- percentual } \\
\text { de encurtamento circunferencial; ns- não significativo. }\end{array}$} \\
\hline
\end{tabular}

ro de pacientes com piora clínica e que necessitaram de internação no G-II, demonstrando que a permanência da libação alcoólica é danosa ao miocárdio.

Assim, apesar do desejo inicial dos participantes do estudo, a abstinência só foi obtida em $50 \%$ dos pacientes com CMA e disfunção ventricular moderada, porém no grupo onde foi alcançada, obtivemos melhor evolução (inalterados + melhoras $=90 \%$ ), com redução do DDVE e do DSVE, devendo ser perseguida mesmo nos pacientes com disfunção ventricular moderada.

\section{Discussão}

A CMA é mais freqüente entre os homens, de 30 a 55 anos, e que consomem mais do que $80 \mathrm{~g}$ etanol $/ \mathrm{dia}^{8}$. Nossa população, de acordo com a literatura, era formada, predominantemente, por negros (55\%), refletindo nossas características de distribuição racial, que apresenta 68635438 $(45,04 \%)$ habitantes pardos ou negros ${ }^{14}$, porém menor do que a referida por Demakis e $\mathrm{col}^{9}$, de 82,45\%, em Chicago.

Discute-se, qual o tempo e a dose mínima de álcool, que provoca agressão ao miocárdio, e se haveria algum grau de predisposição genética na sua caracterização. $\mathrm{O}$ utilizado, mais amplamente, é o do consumo maior do que $80 \mathrm{~g}$ de etanol/dia durante os últimos $10 \operatorname{anos}^{8}$, entretanto, doses menores como $60 \mathrm{~g}$ etanol/dia em períodos mais prolongados (média 25 anos), também podem ser responsáveis pela agressão ${ }^{15}$. Como a dose do álcool depende do tempo em que foi consumido de maneira abusiva, foi possível calcular a dose média responsável em acarretar cardiomiopatia dilatada, como sendo de $250 \mathrm{~kg}$ de etanol durante todo o tempo de libação do indivíduo ${ }^{16}$. O tempo de abuso do álcool em nossa casuística foi de 51 a 112 meses, inferior ao médio (10 anos ou 120 meses) referido na literatura, talvez devido às intercorrências que o álcool possa ocasionar no estado nutricional ou na atividade imunológica, provocando maior grau de agressão miocárdica, em indivíduos que habitam região de clima temperado, sem maiores modificações sazonais.

A predisposição genética é encontrada na doença hepática dos alcoólatras ${ }^{17}$ e relacionada com a presença do antígeno $\mathrm{B}_{6}$ nos pacientes com cardiomiopatia dilatada que consomem álcool de modo exagerado, sugerindo a existência da predisposição genética na agressão ao coração ${ }^{18}$. Em nenhum dos nossos casos tínhamos história familiar de CMA, entretanto, a sua exclusão não afasta a possibilidade da existência de predisposição genética ao álcool, o que não foi alvo do nosso estudo.

A casuística que alcançamos foi pequena, porém, somente atingida após busca exaustiva. Aparentemente, seria muito fácil conseguirmos pacientes com o perfil requerido para o trabalho, porém ao apresentarmos seu desenho, encontrávamos grande relutância na sua aceitação, motivado pela exposição que os pacientes teriam de seus problemas pessoais, que contribuíram para o consumo abusivo do álcool, além da insegurança na participação de terapia de apoio em grupo. Apesar do desejo inicial em participar do 
programa, somente $11(55 \%)$ pacientes, completaram os 12 meses propostos, sendo que somente $8(72,72 \%)$ atingiram a abstinência, tendo três completado o programa, no entanto, sem alcançar o seu objetivo. Entre os $9(45 \%)$ que abandonaram a psicoterapia, $2(10 \%)$ atingiram o objetivo, por terem aderido a seita religiosa onde a abstenção era, também, meta a ser alcançada. Durante o apoio, foram trabalhados a valorização individual e a auto-estima, além da estima familiar e do papel da sociedade e do cidadão.

Na história natural traçada por Demakis e col ${ }^{9}$, em 57 pacientes com CMA, acompanhados por período médio de 40,5 meses, observaram a existência de três tipos de evolução: uma que melhorou o quadro clínico (15 pacientes), onde a maioria 73,3\% (11/15) alcançou a abstenção do álcool; outra, que permaneceu inalterada (12 pacientes) onde somente $25 \%$ (3/12) atingiram a abstinência e o $3^{\mathrm{a}}$ (30 pacientes) que piorou e apenas 13,3\% (4/30) casos tinham abandonado o hábito de consumir álcool. Assim constataram que $69,42 \%$ (39/57) continuaram a consumir álcool e apenas $10,25 \%$ (4/39) apresentaram melhoras clínicas, em contraste com $31,58 \%$ (18/57) onde a abstinência foi alcançada e que $61,1 \%$ (11/18) apresentaram melhoras na função ventricular. Já entre os 64 pacientes, acompanhados por quatro anos e relatados por Arroyo e Regan ${ }^{19}$, apenas 34,3\% (22/64) alcançaram abstinência e $46 \%$ apresentaram melhoras da cardiopatia, $36 \%$ permaneceram inalterados, $9 \%$ piorarame $9 \%$ faleceram. Entre os $42(65,7 \%)$ que não lograram abstenção, $5 \%$ melhoraram, $28 \%$ permaneceram inalterados, $10 \%$ pioraram e $57 \%$ evoluíram para o óbito, nos quatro anos de observação.

Nossos achados foram melhores do que os referidos por Demakis e col ${ }^{9}$ e Arroyo e Regan ${ }^{19}$, pois alcançamos abstinência em $50 \%$ da população estudada, enquanto aqueles autores atingiram $31,58 \%$ e $34,3 \%$, respectivamente, o que foi devido ao fato dos nossos pacientes terem manifestado, previamente, seu desejo de participar do grupo de apoio.

A abstinência alcoólica pode melhorar e contribuir para o desaparecimento dos sintomas cardíacos, com reversão da depressão miocárdica e do tamanho do coração ${ }^{20-23}$, entretanto, pode ser observado defeito de perfusão miocárdica ao esforço avaliado por meio da tomografia com tálio-20123.

Considerando melhoras $(n=3)$ e inalterados $(n=6)$, no grupo de abstinência em relação a melhoras $(n=1)$ e inalterados $(n=4)$, no grupo que persistiu consumindo álcool, os pacientes que lograram atingir o objetivo do estudo, quando avaliados por meios clínicos e por exames complementares em 12 meses, tiveram melhor evolução. Isto nos faz per- sistir na indicação da abstinência do álcool, mesmo nos portadores de disfunção moderada, pois ela é fundamental para modificar a história natural dessa agressão. Tivemos um caso de paciente que, mesmo, continuando na terapia de apoio, não atingiu a abstenção, porém relatou redução no volume diário de álcool, tendo apresentado melhoras clínicas e dos exames complementares, concordando com a proposta de Konstan e col ${ }^{13}$ que permite o consumo de dose única de etanol/dia $(<28 \mathrm{~g})$ naqueles onde o vício não pôde ser totalmente vencido.

Entre os seis pacientes que tiveram piora do quadro clínico, cinco foram hospitalizados durante o período do estudo, sendo a maioria (4/5) entre os que persistiram abusando do álcool. O único paciente internado entre os que atingiram a abstinência, era portador de disfunção, que não foi modificada pelo afastamento do álcool, provavelmente devido ao grau de fibrose apresentado.

Foi concluído que o papel da abstinência também era notado quando a CMA era associada a outras cardiopatias. Venkatran e col ${ }^{24}$ observaram a melhora na associação com doença arterial coronária trivascular, comprovada pelo aumento da fração de ejeção de 11 para $61 \%$ obtida por radionuclídeo após oito meses de abstenção. Stöllberg e Finsterer ${ }^{25}$ observaram a reversão da cardiomiopatia dilatada para hipertrófica, em jovem de 35 anos, consumindo álcool durante 13 anos, e cuja abstinência de um ano modificou, de maneira marcante o tipo da cardiomiopatia. Em nossa população não tínhamos nenhum paciente com associação de cardiopatias.

Os alcoólatras podem apresentar redução da ingesta de vitamina B1 (tiamina) que pode ocasionar a chamada tintura beribérica, que é a carência sem os sintomas e sinais característicos do beribéri e que, rapidamente, desaparecem com a reposição desta vitamina. Cerca de $15 \%$ dos alcoólatras assintomáticos apresentam moderada deficiência desta vitamina ${ }^{26}$ e que $10 \%$ dos pacientes com IC só melhoram o quadro congestivo após a adição da tiamina ao esquema terapêutico ${ }^{27}$. Para evitar esta contaminação, nossos pacientes, durante o período do programa de apoio, não fizeram uso de complementação vitamínica a fim de não modificar os achados da abstinência.

Cremos, que apesar dos custos serem elevados para a realização de programas de apoio a alcoólatras, eles devessem ser estimulados, pois seu efeitos são evidentes quanto ao papel de regressão da disfunção miocárdica, do número de hospitalizações por descompensação cardíaca e por propiciar maior integração familiar, social e comportamental do cidadão. 


\section{Referências}

1. Walshe WH - The Practical Treatise on the Physical Diagnosis. $4^{\text {th }}$. London: Smith and Elder, 1873.

2. Steel G - Heart failure as a result of chronic alcoholism. Med Chron Manchester 1893; 18: $1-5$.

3. Eliaser M, Giansiracusa F - The heart and alcohol. Calif Med 1956; 84: 234-7.

4. Evans W- The electrocardiogram of alcoholic cardiomyopathy. Br Heart J 1959; 21: 445-56.

5. Brigden WS, Robinson J - Alcoholic heart disease. Br Med J 1964; 2: 1283-9.

6. Benchimol AB, Schelesinger P, Barbosa $\mathrm{F}^{\circ} \mathrm{J}$ - Cardiomiopatia alcoólica. J Br Med 1967; 13: 111- 6.

7. Kasper EW, Agema WRP, Hutchins GM, Deckers JW, Hare JM, Baughman KL The cause of dilated cardiomyopathy: a clinicopathologic review of 673 consecutive patients. J Am Coll Cardiol 1994; 23: 586-90.

8. Burch GE, Gilles TD - Alcoholic cardiomyopathy: concept of disease and its treatment. Am J Med 1971; 101: 461-4.

9. Demakis JG, Proskey A, Rahimtoola SH et al - The natural course of alcoholic cardiomyopathy. Ann Inter Med 1974; 80: 293-7.

10. Schlesinger $\mathrm{P}$, Benchimol AB, Barbosa $\mathrm{F}^{\circ} \mathrm{J}$, Benchimol CB - Cardiomiopatia alcoólica. In: Benchimol AB, Schlesinger P (ed) - Enciclopédia Médica Brasileira - Cardiologia. Rio de Janeiro: Guanabara Koogan, 1978: 1-15.

11. Regan TJ - Alcohol and cardiovascular system. JAMA 1990; 264: 377-81.

12. Silva MAD - Miocardiopatia dilatada. In: Silva MAD (ed) - Doença do Miocárdio. São Paulo: Sarvier, 1995: 96.

13. Konstam M, Dracup K, Baker D et al - Heart failure: management of patients with left-ventricular systolic dysfunction. Quick reference guide for clinicians $\mathrm{n}^{\circ} 11$. AHCPR Publication No. 94-0613. Rockville, MD: Agency for Health Care Policy and Research, Public Health Service. US Department of Health and Human Services, Washington, DC, 1994.

14. Anuário Estatístico do Brasil/Instituto Brasileiro de Geografia e Estatística. Volume 56, Seção 2-Saúde, 1996: 2-109 - 2-137.

15. Gillet C, Julliere Y, Pirollet P et al - Alcohol consumption and biological markers for alcoholism in idiopathic dilated cardiomyopathy: a case-controlled study. Alcohol Alcohol 1992; 27: 353-8.
16. Richardson PJ, Wodak AA - Alcohol-induced heart muscle disease. In: Symons C, Evans T, Mitchell AG (eds) - Specific Heart Muscle Disease. Bristol: Wright PSG, 1983; 99-122.

17. Saunders JB, Williams R - The genetics of alcoholism: is there an inherited susceptibility to alcohol-related problems? Alcohol Alcohol 1983; 18: 189-218.

18. Fischbein L, Sachs RN, Geay D et al - Study of HLA system A and B antigens in dilated cardiomyopathy associated with alcoholism. Arch Mal Coeur 1987; 80: 1171-5.

19. Arroyo LH, Regan TJ - Ethanol and Heart. In: Topol E (ed) - Textbook of Cardiovascular Medicine. Philadelphia: Lippincott-Raven Publishers, 1998: 219-29.

20. Schwartz L, Sample KA, Wigle ED - Severe alcoholic cardiomyopathy reversed with abstention from alcohol. Am J Cardiol 1975; 36: 963-6.

21. Marette P, Toussaint M, Spaulding C et al - Myocardiopathie dilatée avec alteration profonde de la fontion gauche chez l'alcoolique, totalement reversible: à propos de deux cas. Arch Mal Coeur 1990; 83: 1843-7.

22. Mansourati J, Forneiro I, Genet L, Le Pichon J, Blanc JJ - Regression d'une cardiomyopathie dilatée chez un ethylique chronique apràs arrêt de l'íntoxication. Arch Mal Coeur 1990; 83: 1849-52.

23. Juilliere Y, Gillet C, Danchin N et al - Abstension from alcohol in dilated cardiomyopathy: complete regression of the clinical disease but persistence of myocardial perfusion defects on exercise thallium-201 tomography. Eur J Nucl Med 1990; 17: 279-81.

24. Venkatram N, Brown EJ, Timson CR, Patcha R - Reversal of alcoholic cardiomyopathy in patient with severe coronary artery disease. Chest 1993; 104: 626.

25. Stöllberger C, Finsterer J - Reversal of dilated to hypertrophic cardiomyopathy after alcohol abstinence. Clin Cardiol 1998; 21: 365-7.

26. Neville JN, Eagles JA, Sampson G et al - The nutritional status of alcoholics. Am J Clin Nut 1968; 21: 1329-40.

27. Pinn G, Bovet P - Alcohol-related cardiomyopathy in Seychelles. Med J Aust 1991; 155: 529-32. 